\title{
Co existing Abdominal Tuberculosis and Mucinous Adenocarcinoma of Colon: Coincidence or Causal Nexus?
}

\author{
${ }^{1}$ SM Ikhwan, ${ }^{1}$ LS Bob, ${ }^{2}$ AAM Zin, ${ }^{1}$ Z Zaidi \\ ${ }^{1}$ Department of Surgery School of Medical Sciences Universiti Sains Malaysia Health Campus 16150 Kubang \\ Kerian Kelantan, Malaysia \\ ${ }^{2}$ Department of Pathology School of Medical Sciences Universiti Sains Malaysia Health Campus 16150 Kubang \\ Kerian Kelantan, Malaysia
}

\begin{abstract}
Abdominal tuberculosis and colonic carcinoma at this part of the world are very common but coexisting abdominal tuberculosis and mucinous adenocarcinoma is unusual and is considered a rare entity. We had a patient who was diagnosed as splenic flexure carcinoma with lung metastasis preoperatively.

Due to bleeding tumour, we proceeded with tumour debulking, gastrojejunostomy and creation of endstoma. Histopathology showed features of both mucinous adenocarcinoma and abdominal tuberculosis. Literature was reviewed and found that very few of such cases have been reported. Most of these cases shared some common features with predominant involvement of younger age group, right sided colonic involvement and predominantly a mucinous adenocarcinoma.

The cause-effect relationship between these two conditions has been discussed and debatable. We postulated that this patient most likely had colonic carcinoma initially, then subsequently infected with tuberculosis. However, there is still no definitive cause-effect relation could be ascertained and lots of study are needed to detect or explain the association between these two different pathologies.
\end{abstract}

\section{Introduction}

Abdominal tuberculosis and colonic carcinoma are very common conditions but the sites of predilection differ with tuberculosis being more common in the ileocaecal junction or terminal ileum and carcinoma in the distal large bowel or left sided colon; and both occurring at same site is a very rare entity.

Although there are few cases have been reported, so far no cause-effect relation could be defined. The objective of writing this case report is to report the rare case and reviewing the literature, aiming to answer whether both the pathologies just merely co-incidence or is there any cause-effect relation between these two conditions.

\section{CASE REPORT}

A 47-year old gentleman was referred to us from a district hospital with 1 month history of watery diarrhea. The frequency was $\sim 5$ times daily and there was no blood or mucus in the stool. It was associated with colicky abdominal pain and tenesmus. He also had constitutional symptoms such as anorexia and significant loss of weight (20kg over the last 5 months). He was symptomatic of anemia with lethargy, palpitations and occasionally shortness of breath. In fact, he was admitted twice to the district hospital 4 months and 2 weeks ago preceding current admission for symptomatic anemia and blood transfusion was given. Hematological investigations showed microcytic hypochromic anemia.

He had no chronic medical illness or any history of tuberculosis. He denied history of night sweat, chronic cough or any contact with pulmonary tuberculosis patient. Also, he had no previous history of surgery. Clinically he was a middle aged gentleman, cachexia, pale, weight $37 \mathrm{~kg}$, afebrile, blood pressure of $100 / 70 \mathrm{mmHg}$ and heart rate of $78 / \mathrm{min}$. There was no cervical lymph node palpable. Abdomen was soft, not distended, non-tender and no mass was palpable, hernia orifices were intact and no hepato-splenomegaly; but there was presence of ascites. Digital rectal examination revealed empty rectum and no mass palpable. Other systemic examinations including cardiovascular and lungs were normal. There was minimal pedal edema bilaterally.

Biochemically, his haemoglobin was $8.6 \mathrm{~g} / \mathrm{dl}$, total white cells 13,000 , potassium $3.0 \mathrm{mmol} / \mathrm{L}$, albumin $18 \mathrm{~g} / \mathrm{dL}$. Liver enzymes were normal. Colonoscopy was performed showing tumour at $70 \mathrm{~cm}$ from anal verge and unable to negotiate the scope further. Biopsies were taken from the tumour and histopathologically confirmed malignancy with mucinous adenocarcinoma.

Chest X-ray (Fig. 1) was normal. Computed tomography (CT) of thorax, abdomen and pelvis revealed a circumferential heterogenouly enhancing mass involving distal transverse colon, splenic flexure and proximal part of descending colon (length of involved segment $\sim 15 \mathrm{~cm}$ ); poor plane demarcation with greater curvature of the stomach, jejunum and tail of pancreas (Fig. 2). There was adjacent mesenteric fat streakiness and presence 
of multiple mesenteric lymph nodes with the largest measured $1.0 \mathrm{~cm}$. There were multiple lung nodules in the medial segment of middle lobe, left posterobasal segment, right laterobasal, mediobasal and posterobasal segments.

In view of $\mathrm{CT}$ finding with poor plane demarcation of the tumour with greater curvature of the stomach, oesophago-gastric-duodeno-scopy (OGDS) was performed which revealed only multiple D1 and D2 Forrest III ulcers, with no mass was seen.

In the ward, he was given blood transfusion and total parenteral nutrition to build up his nutrition status pre-operatively. Unfortunately, the patient experienced massive per-rectal bleeding in the ward. He was then subjected to emergency laparotomy. Intraoperatively, there was straw-coloured ascetic fluid of 700cc; a splenic flexure tumour (Fig. 3 \& Fig. 4) very adherent to the DJ flexure, part of greater curvature of the stomach, with omentum plastered to it; and posteriorly adhered to the Gerota's fascia. There were no peritoneal seedlings but presence of small nodules in the liver. Tumour debulking which included en-bloc resection of tumour at splenic flexure with greater curvature of the stomach, tail of the pancreas and fourth part of the duodenum; gastrojejunostomy and creation of end-stoma was performed for him.

Histopathologically revealed mucinous adenocarcinoma (Fig. 5) of the splenic flexure tumour, infiltrating into the serosal layer of the descending colon, serosal layer of the stomach and up to mucosal layer of the jejunum. There were foci of caseous necrosis with Langhans type giant cells seen in the serosal layer of descending colon and jejunum. There were 16 lymph nodes identified and all of them showed caseous necrosis with occasional Langhans type multinucleated giant cells seen (Fig. 6). Ziehl-Neelsen staining was positive for acid fast bacilli (Fig. 7). All surgical margins were free from tumour. A diagnosis of mucinous adenocarcinoma associated with tuberculosis was made.

Post operatively, he was monitored in the intensive care unit. However, he was hemodynamically unstable, requiring inotropes and succumbed to his illness two days after the operation.

\section{Discussion}

The most common malignancy of the gastrointestinal tract is colorectal carcinoma. The mean age at presentation is around 70-75 years; it has a negligible overall predominance of female patients. Forty to fortyfive percent of colorectal cancers are located in the rectum (10-15\%) or sigmoid colon (40\%) and $25-35 \%$ in the caecum or ascending colon whereas the remainder is equally distributed through the rest of the colon [3]. Regarding histological variant, the predominant is adenocarcinoma (90-95\%), followed by mucinous adenocarcinoma (10\%) and others like signet ring cell and small cell carcinoma [3]. Mucinous adenocarcinoma has the worst prognosis as the pockets of mucin can mechanically dissect along the planes of the bowel wall, facilitating the spread of malignancy.

Abdominal tuberculosis is infection of peritoneum, hollow or solid abdominal organs by Mycobacterium. Gastro-intestinal tuberculosis is commoner among the young adults and there is also a slight female preponderance [3]. Our case however, was a young male. After lymphatics, genitourinary system, bone \& joint, miliary tuberculosis and meninges, the gastrointestinal tract is the sixth frequent site of extra-pulmonary tuberculosis. Tuberculosis bacteria reach the gastrointestinal tract via haematogeneous spread, ingestion of infected sputum or direct spread from infected contiguous lymph nodes and fallopian tubes [4]. The site of predilection of abdominal tuberculosis is dictated by several factors. Due to physiological status, increase rate of fluid and electrolyte absorption, minimal digestive activity and an abundance of lymphoid tissue, the ileocaecal region is the most common site of gastrointestinal tuberculosis. The frequency of bowel involvement decreases as one proceeds both proximally and distally from the ileocaecal junction [4].

The association of tuberculosis and cancer has since been recorded in most organs by various authors. Carcinoma in different parts of the colon with intestinal tuberculosis have been reported by Paustian [5]. In New York, Kaplan et al. found tuberculosis complicating neoplastic disease in only 4 out of 6472 patients with carcinoma of the colon, accounting for a prevalence of 6/10,000 [6].Some Indian authors have proposed that the association of carcinoma of colon and tuberculosis is co-incidental. This is based on the argument that the cases of co-existing tuberculosis and carcinoma are very few, compared to the high incidence of abdominal tuberculosis in India [1]. This may be true in some cases particularly when the colonic tumour occurs at a site distant from the tubercular focus. Having said that, to put the simultaneous occurrence of the two different pathologies at the same site down to mere co-incidence is by far too simplistic. Whenever two different pathologic lesions are associated closely, the aetiological relationship between two diseases is a matter of debate.

In a large number of reports in the literature of the co-existing tuberculosis and colonic carcinoma, the malignant lesion is merely described as a cancer or carcinoma without specifying the precise histopathological type [1]. Tanaka et al analyzed 26 cases of co-existing tuberculosis and colonic carcinoma and reported that females predominated by a ratio of 17.9 in such cases; the tumor was found in the right side of the colon in 17 
out of 26 cases and thirdly, the tumor showed a well differentiated adenocarcinoma with a tendency to produce mucin [2].

Jain B.K et al. reported 4 cases of co-existing colonic carcinoma and abdominal tuberculosis; the tumour involved the right colon in 3 out of 4 cases and the distal transverse colon in the $4^{\text {th }}$ case. The lesions co-existed at the same site in two of the cases [1]. This case differs from them as such our patient was a gentleman and the colonic carcinoma was at the left side, except that it was mucinous type.

Chronic inflammatory mucosal damage initiating a sequence of metaplasia and dysplasia results in neoplastic change. This is evidenced by some diseases like ulcerative colitis, Crohns disease, and schistosomiasis predispose to malignancy. Thus, it may be postulated that the ulcerative lesions of intestinal tuberculosis are precursors of carcinomas and this possibility was suggested by Japanese researchers [2]. These carcinomas arose as a result of repeated insults by way of erosions, ulceration, and consequent regeneration.

Having mentioned that, another view is that carcinoma may facilitate the entry of tubercle bacilli with development of secondary infection. This view might be correct in only those cases in which tuberculosis occurs at an unusual site like left sided colon or in older group of patients. It is universally accepted that factors that disturb host immunity increase susceptibility to active tubercular infection. Severe weight loss or malnutrition related to an advanced neoplastic disease is such a factor. Conceivably invasion of a dormant tubercular lesion by carcinoma could lead to activation and endogenous reinfection. Locally produced tumour peptides or antigens may also upset the milieu of a granuloma and allow the tuberculous organisms to proliferate [6]. This might be true in our patient with his middle age group and involvement of left sided colon.

The best treatment in this case is difficult to decide as pre-operative diagnosis of such a coexisting dual different pathology is virtually impossible [7].

\section{Conclusion}

This case highlights the need and importance of complete evaluation of patients with iron deficiency anemia. Pertaining to the abdominal tuberculosis and colonic carcinoma, there is still no definitive cause-effect relation could be ascertained and lots of study is needed to detect or explain the association between these two pathologies.

\section{References}

[1]. Jain, B.K., et al., Coexisting tuberculosis and carcinoma of the colon. Aust N Z J Surg, 1991. 61(11): p. 828-31.

[2]. Tanaka, K., et al., [A case of colonic carcinoma associated with intestinal tuberculosis, and an analysis of 26 cases reported in Japan]. Gan No Rinsho, 1987. 33(9): p. 1117-23.

[3]. M. Husain, P.S., S. Khan, N. Chauhan, S. Kishore, Co-existing Abdominal Tuberculosis and Colon Carcinoma: Care Report with Review of Literature, in The Internet Journal of Surgery. 2007.

[4]. Sharma, M.P. and V. Bhatia, Abdominal tuberculosis. Indian J Med Res, 2004. 120(4): p. 305-15.

[5]. Paustian, F., Tuberculosis of intestine. 3rd ed. Gastroenterology. Vol. 2. 1976: In: Bockus HL, editor. Philadelphia, London: W.B. Sauder Co. p.773.

[6]. Kaplan, M.H., D. Armstrong, and P. Rosen, Tuberculosis complicating neoplastic disease. A review of 201 cases. Cancer, 1974. 33(3): p. 850-8.

[7]. Kaushik, R., R. Sharma, and A.K. Attri, Coexisting tuberculosis and carcinoma of the colon: a report of two cases and a review of the literature. Trop Gastroenterol, 2003. 24(3): p. 137-9.

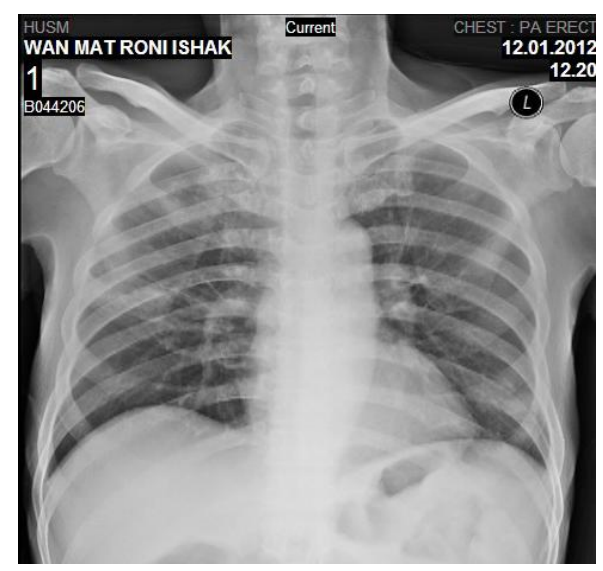

Fig. 1: Normal Chest X-ray 


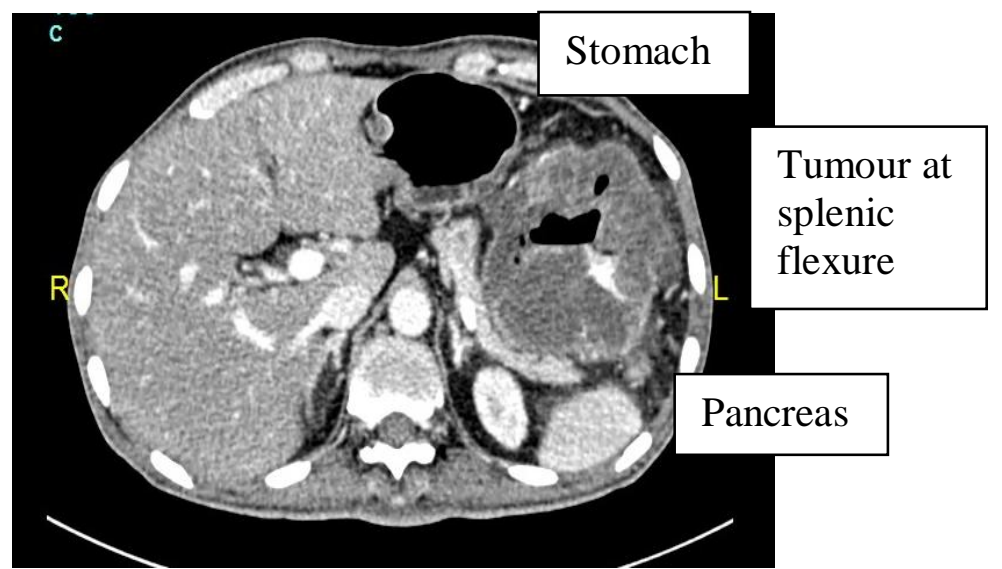

Fig. 2: CT shows poor plane demarcation of the tumour with greater curvature of the stomach and tail of pancreas

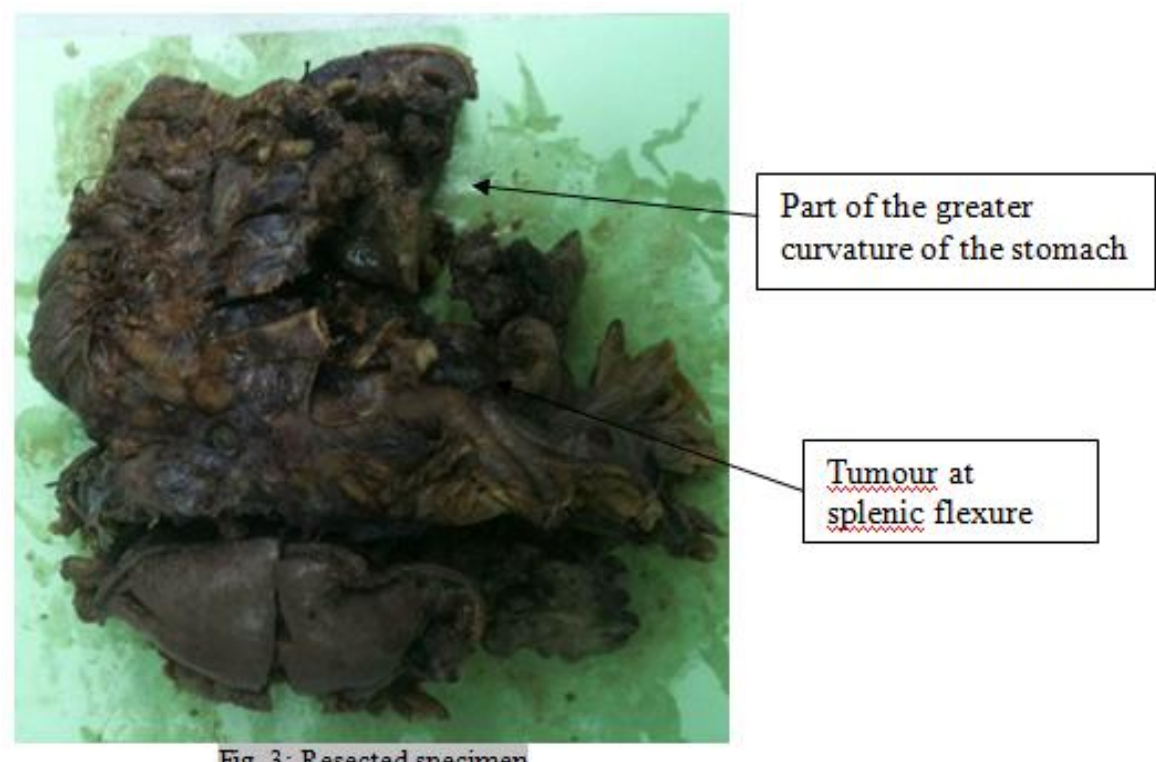

Fig. 3: Resected specimen

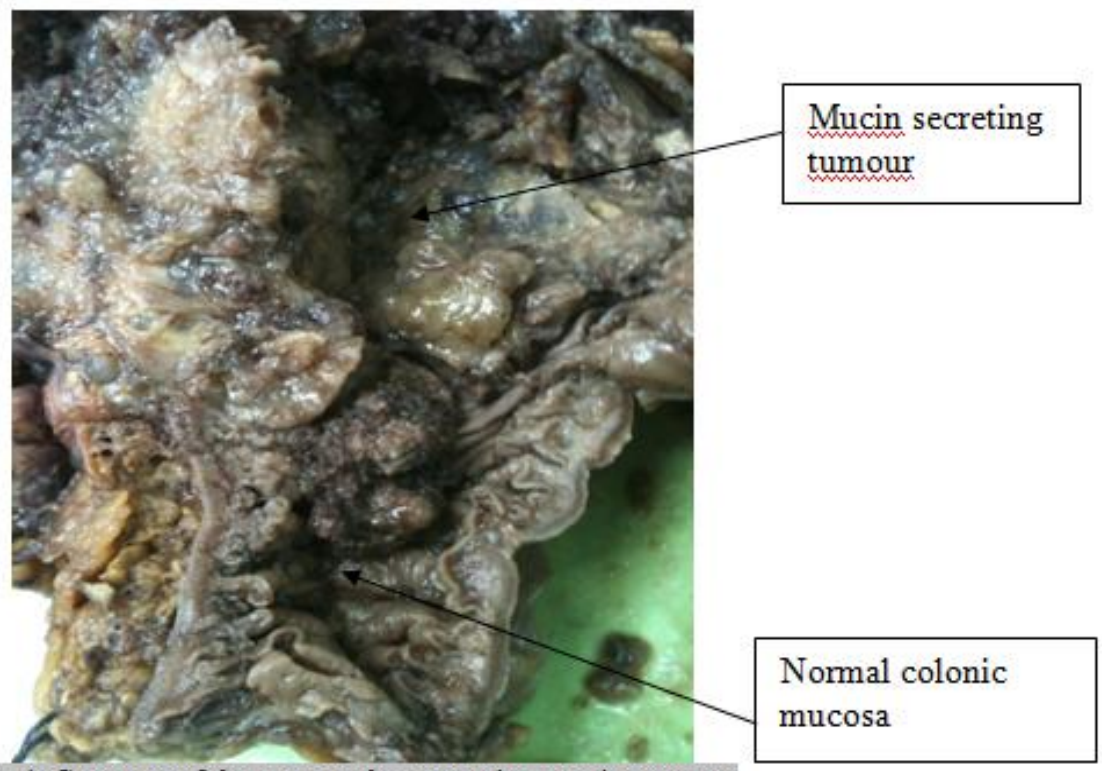

Fig. 4: Cut open of the tumour shows mucin-secreting tumour 


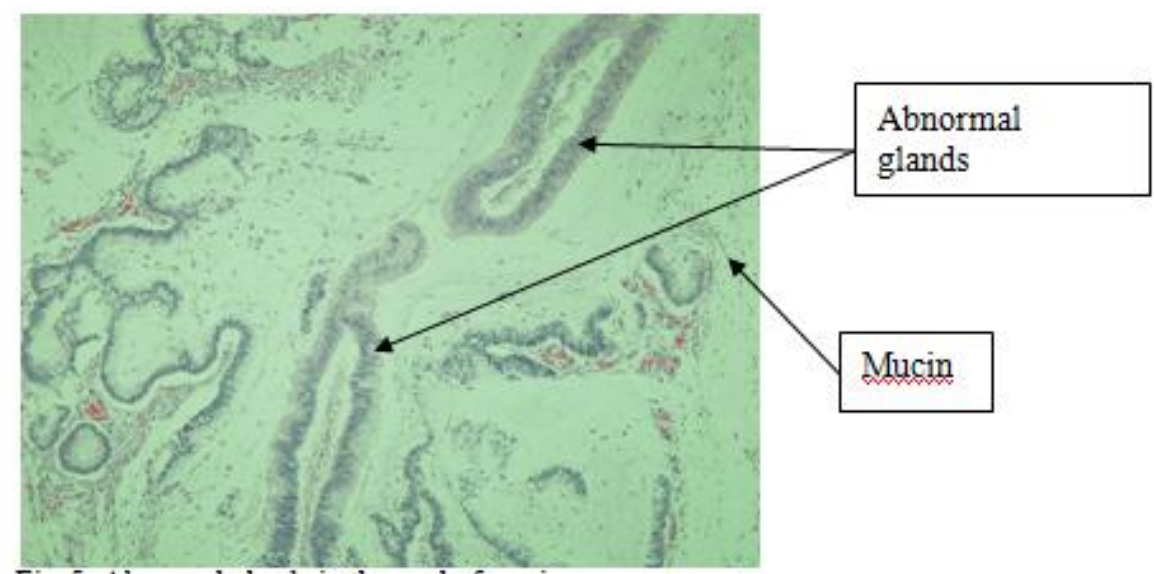

Fig. 5: Abnormal glands in the pool of mucin

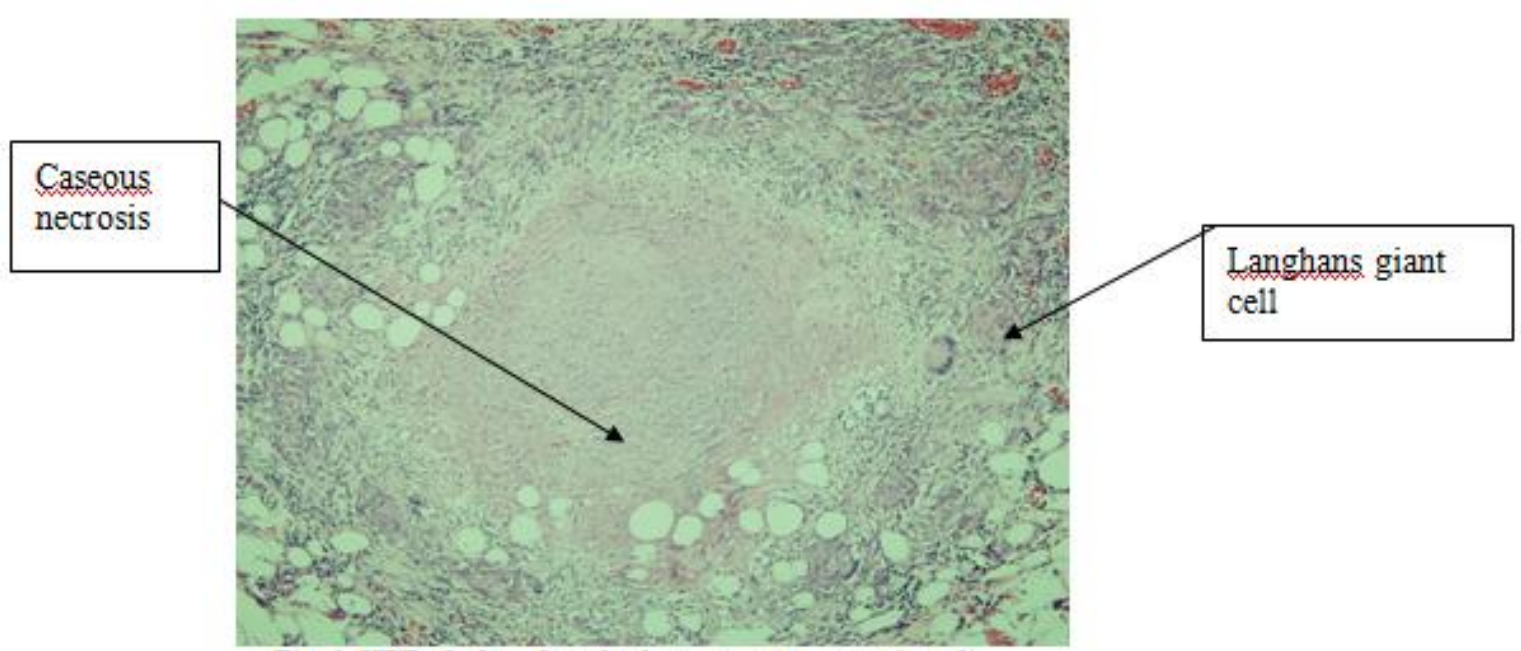

Fig. 6: HPE of a lymph node shows caseous necrosis and

presence of Langhans giant cell

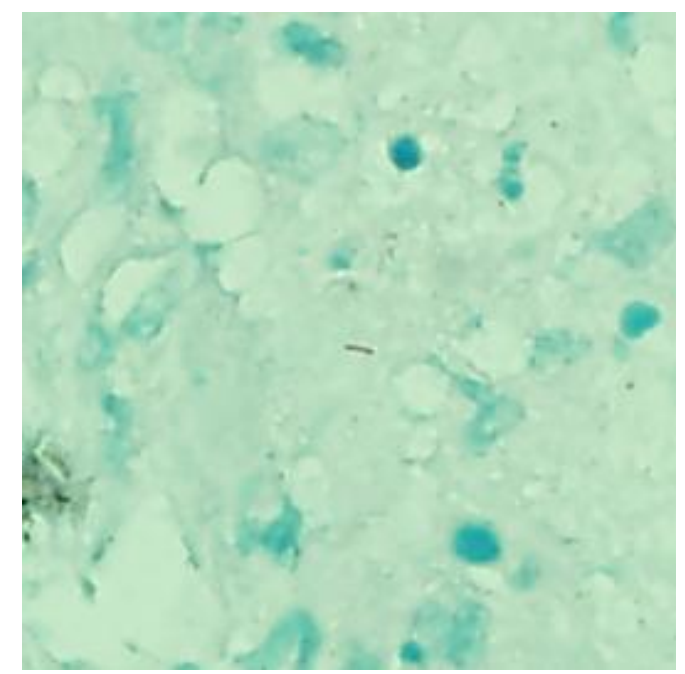

Fig. 7: Ziehl-Neelsen staining shows positive acid fast bacilli 